\title{
Modal and transition dwell time computation in switching systems: a set-theoretic approach
}

\author{
Franco Blanchini ${ }^{1, a}$ Daniele Casagrande ${ }^{b}$ Stefano Miani ${ }^{b}$ \\ ${ }^{a}$ Dipartimento di Matematica e Informatica, Università degli Studi di Udine, Via delle Scienze 208, 33100 Udine, Italy \\ ${ }^{\mathrm{b}}$ Dept. of Electrical, Managerial and Mechanical Engineering, University of Udine, Italy
}

\begin{abstract}
We consider a plant the dynamics of which switch among a family of systems. Each of these systems has a single stable equilibrium point We assume that a constraint region for the state is assigned and we consider the problem of finding suitable limitations on the commutation speed in order to avoid constraints violations, even in the absence of state measurements. We introduce the concepts of modal and transition dwell times which lead to the definition of a dwell time vector and dwell time graph (represented by a proper matrix), respectively. The former imposes a minimum permanence on a discrete mode before commuting, the latter imposes the minimum permanence time on the current mode before switching to a specific new one. Both dwell time vector and dwell time graph, can be computed via set theoretic techniques. When the systems share a single equilibrium state, stability can be assured as a special case. Finally, under the assumption of affine dynamics, non-conservative values are achieved.
\end{abstract}

Key words: Switching systems; Dwell time; Constraints; Stability.

\section{Introduction}

It is well established that a fast switching among a family of stable dynamical systems may lead to instability. This phenomenon is peculiar of plants in which the dynamics of the family are quite different. Stability under arbitrary switching requires a "common feature", beyond individual stability, which is known to be the existence of a common Lyapunov function for all the dynamics $[17,16]$, a quite restrictive condition.

Consequently, when this condition is not satisfied, it is of interest to know under which restrictions on the switching sequence stability is preserved; in particular, the concept of dwell time plays a fundamental role $[19,20]$. The dwell time can be defined as the minimum time interval in which the system is forced to stay in a given logic state, namely the smallest interval between two consecutive switchings and it is an important parameter for stability. The notion of dwell time is important both in the case of uncontrolled (exogenous) switchings and in the case of switchings produced by a supervisor [22]. According to a terminology previously introduced [3], we refer to the former as switching systems

\footnotetext{
1 Corresponding author. Email: blanchini@uniud.it

2 A preliminary version of this paper was submitted to CDCCCC, Shanghai, 2009
}

and the latter as switched systems.

It is interesting to notice that the stability under dwell time admits a Lyapunov characterization; however, in this case multiple Lyapunov functions must be introduced ${ }^{3}$. Some results have very recently been proposed in the literature to compute lower bounds for the dwell time assuring stability [10][6][2]. In particular, some contributions aim at finding the optimal dwell time [8] [9]. In some cases, a restriction on the average commutation interval is more reasonable than a rigid lower bound on the commutation interval. This idea led to the concept of average dwell time for which computable lower bounds have been proposed [12]. Several notions of dwell time have been discussed [13], stability results being proposed mainly by means of multiple Lyapunov functions [7]. From the existing surveys [21][2] and monographs [16][22] it is apparent that there are several unsolved problems in the computation of proper lower bounds for the dwell time to assure stability or, quite harder, the computation of its minimum value, typically possible only in special cases [9].

In this paper we propose a set-theoretic approach to solve the problem of establishing limits on the commutation sequence. The work is characterized by two main features. First, we

\footnotetext{
3 See [21] section 5.2.
} 
consider not only stability but also safety problem, which is the problem of keeping the state in an assigned region and eventually making the trajectory convergence to some, not necessarily unique, equilibrium point. This is a more general problem, being switching stability a special case when the equilibrium is unique. Second, we do not consider a single dwell time but several values for the dwell time which depend on the logic state of the system. We show that these values can be computed via set-theoretic methods and in some cases non-conservative bounds can be evaluated.

In details, we consider a finite family of stable dynamics, each associated with its own equilibrium point, and we assume that an admissible region is assigned. Then the main results can be summarized as follows.

- We introduce the concept of modal dwell time, leading to a dwell time vector and of transition dwell time, leading to the dwell time graph. The dwell time vector imposes, at each time, a minimum permanence on the current discrete mode. The dwell time graph has weighted arcs so that the corresponding transitions are enabled only if the proper time has elapsed.

- We show how dwell time vector and dwell time graph can be computed via set-theoretic methods.

- In the case of affine dynamics, the computation involves polytopes. Necessary and sufficient conditions are achieved to recover the largest region of initial states.

- Stability is faced as a special case in which the dynamics share the same equilibrium.

- As an application, the switching limiter, namely a device which avoids constraint violations without requiring state measurement, is proposed.

\subsection{Motivation}

The main motivation of the work consists in the following problem. Suppose that the switchings between the different modes of a family of dynamical systems occur according to a switching sequence $\hat{\sigma}$. If such a switching system is not guaranteed to be constraint-violation-free (or even stable) under arbitrary switching sequences, then a switching supervisor, namely a device which limits the switching sequence, may be designed (see Figure 1). Such a device basi-

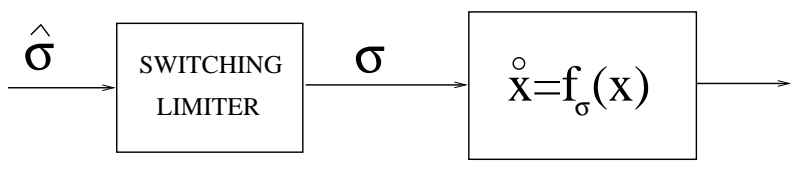

Fig. 1. The switching limiter.

cally replaces the original switching signal $\hat{\sigma}(t)$ with a new one $\sigma(t)$ which prevents the system from being in danger. The idea of a switching supervisor has already been proposed [19] [20] [1]; such a device is important in the presence of constraints [5], in gain scheduling problems [18] or to improve performance [15] [23] [6]. Most of the works available in the literature involve Lyapunov functions and invariant regions. However, measurements from the system are necessary, often including accurate estimation of the state. On the contrary, we assume a-priori stability and, in turn, we achieve a device which does not require state estimation, since the inclusion in the proper "safe set" is assured by construction provided that the prescribed dwell time limits are respected.

\section{Problem statement and basic definitions}

\subsection{Notation}

Throughout the paper, script letters (such as $\mathscr{A}, \mathscr{B}, \ldots$ ) denote sets; int $\mathscr{A}$ denotes the interior of set $\mathscr{A} ; \mathbb{R}_{+}$denotes the extended set of non-negative real numbers, namely including $+\infty$.

\subsection{Problem statement}

Consider the switched dynamical system

$$
\dot{x}(t)=f_{\sigma(t)}(x(t)),
$$

where $x(t) \in \mathbb{R}^{n}, \sigma(\cdot)$ is a switching signal, namely a piecewise constant function assuming value in a finite set $\Sigma \subset \mathbb{N}$, and $f_{i}$, for $i \in \Sigma$, is an assigned function; without restriction we assume $\Sigma=\{1,2, \ldots, s\}$ and $\sigma$ right-continuous. In addition, the following basic assumption is introduced.

Assumption 1 For any $i \in \Sigma$, the functions $f_{i}$ are locally Lipschitz and each dynamics, corresponding to $\sigma=i$, admits a single equilibrium point $\bar{x}_{i}$. Such an equilibrium is globally uniformly asymptotically stable.

Our goal is to find constraints on the switching sequence in such a way that $x(t)$ belongs to a given set for all $t \geqslant 0$. To this aim we introduce three types of dwell-time as follows.

Definition 2.1 Let $_{0}, t_{1}, t_{2}, \ldots, t_{k}, \ldots$, with $0=t_{0}<t_{1}<t_{2}<$ $\ldots<t_{k}<\ldots$, denote the switching instants.

- We say that $\sigma(\cdot)$ is subject to the dwell time $\tau \in \mathbb{R}_{+}$if for all $k \in \mathbb{Z}_{+}$

$$
t_{k+1}-t_{k} \geq \tau
$$

- We say that $\sigma(\cdot)$ is subject to the modal dwell time $\tau_{j} \in$ $\mathbb{R}_{+}$, associated with the $j$-th mode of system (1) if for all $k \in \mathbb{Z}_{+}$such that $\sigma(t)=j$ for $t \in\left[t_{k}, t_{k+1}\right)$

$$
t_{k+1}-t_{k} \geq \tau_{j}
$$

- We say that $\sigma(\cdot)$ is subject to the transition dwell time $\tau_{i j} \in \mathbb{R}_{+}$, relatively to the commutation from $j$ to $i$, if for all $k$ such that $\sigma(t)=j$ for $t \in\left[t_{k}, t_{k+1}\right)$ and $\sigma\left(t_{k+1}\right)=i$

$$
t_{k+1}-t_{k} \geq \tau_{i j}
$$


For reasons that will soon be clear, we admit that $\tau_{j}=+\infty$ (as well as $\tau_{i j}=+\infty$ ), meaning in this case that no transition is possible from mode $j$ (and, respectively, that no transition is possible from mode $j$ to mode $i$ ).

Now, consider a compact region $\mathscr{X} \subset \mathbb{R}^{n}$ and suppose it has to be regarded as an operating region for $x(t)$, namely a hard constraint for the admissible trajectories. The following question concerning dwell-time constraints is analyzed in the paper.

Problem 2.1 Given a set $\mathscr{X} \subset \mathbb{R}^{n}$, find lower bounds $\bar{\tau}_{j}$ for $\tau_{j}\left(\right.$ or $\bar{\tau}_{i j}$ for $\left.\tau_{i j}\right)$ and proper sets of initial conditions $\mathscr{X}_{i}$, $i \in \Sigma$, such that

$$
x(0) \in \mathscr{X}_{\sigma(0)} \Rightarrow x(t) \in \mathscr{X}, \quad \forall t>0 .
$$

We will also consider a stronger version of the problem in which a common initial condition set is required, namely, $\mathscr{X}_{i}=\mathscr{X}_{0}$ for all $i$.

To have a meaningful solution to the above problem, we make the following assumption.

Assumption 2 The set $\mathscr{X}$ has a non-empty interior, int $\mathscr{X}$, and, for all $i \in \Sigma, \bar{x}_{i} \in$ int $\mathscr{X}$.

To find a solution to Problem 2.1, we exploit the set-theoretic approach, for which the regions introduced next play a fundamental role.

Given a point $x_{0} \in \mathbb{R}^{n}$, let $\Phi_{i}\left(t, x_{0}\right)$ denote the solution at time $t$ corresponding to the $i$-th dynamics and to the initial condition $x_{0}$. Given a set $\mathscr{S} \subset \mathbb{R}^{n}$, let $\Phi_{i}(t, \mathscr{S})$ denote the set of solutions corresponding to the $i$-th dynamics and to an initial condition in the set $\mathscr{S}$ :

$$
\Phi_{i}(t, \mathscr{S})=\left\{\Phi_{i}(t, y): y \in \mathscr{S}\right\}
$$

Definition 2.2 The largest positively invariant set for the system $\dot{x}=f_{j}(x)$, denoted by $\mathscr{P}_{j}$, is the set of all initial states $x_{0} \in \mathscr{X}$ such that $\Phi_{j}\left(t, x_{0}\right) \in \mathscr{X}$, for all $t>0$, namely, the largest set $\mathscr{S}$ for which $\Phi_{j}(t, \mathscr{S}) \subseteq \mathscr{X}$ for all $t$.

In the paper the set

$$
\mathscr{P}=\bigcap_{j \in \Sigma} \mathscr{P}_{j}
$$

will play a fundamental role. Standard results for invariant sets are reported next.

Lemma 2.1 The sets $\mathscr{P}_{j}$ are closed (therefore such is $\mathscr{P}$ ).

Lemma 2.2 The equilibrium points $\bar{x}_{j}$ are in the interior of the forward propagation of the corresponding domain: $\bar{x}_{j} \in \operatorname{int} \Phi_{j}\left(t, \mathscr{P}_{j}\right)$ for all $t \geq 0$.
Proof. We first note that $\bar{x}_{j} \in$ int $\mathscr{P}_{j}$. This is due to the fact that, by assumption $\bar{x}_{j} \in$ int $\mathscr{X}$, and that $\bar{x}_{j}$ is uniformly asymptotically stable. This proves the lemma for $t=0$. Since $f_{j}(x)$ is locally Lipschitz and the solutions are bounded, for any $j$ we have uniqueness of the solution and continuity on the initial (and final) data. Then $\mathscr{P}$ and $\Phi_{j}(t, \mathscr{P})$ are isomorphic. Now $\mathscr{P}$ is a closed set with a non-empty interior. Then a point $x(t)$ of a system trajectory is in the interior of $\Phi_{j}(t, \mathscr{P})$ if and only if originates from a point $x(0)$ in the interior of $\mathscr{P}$. Therefore $\bar{x}_{j}$ cannot be on the boundary. $\diamond$

Remark 2.1 Note that the assumption that $f_{j}(x)$ is locally Lipschitz is crucial in the previous lemma. If we remove the Lipschitz assumption, the system $\dot{x}=-\sqrt{|x|}$ and the interval $[-1,1]$, provide a simple counterexample to the Lemma since any trajectory originating in such an interval converges in finite time to 0 .

An interesting property concerning the sets $\mathscr{P}_{j}$ and $\mathscr{P}$, which is a direct consequence of their definitions, is that they are maximal sets of initial conditions for the permanence of the trajectory in $\mathscr{X}$, as stated in the following result.

Proposition 2.1 The following implications hold.

- $x(t) \in \mathscr{X} \forall t>0$ implies that $x(0) \in \mathscr{P}_{\sigma(0)}$.

- $x(t) \in \mathscr{X} \forall t>0$ and all $\sigma(0) \in \Sigma$ implies $x(0) \in \mathscr{P}$.

According to Proposition 2.1 we call $x(0)$ a feasible initial condition if $x(0) \in \mathscr{P}_{\sigma(0)}$ and safe initial condition if $x(0) \in$ $\mathscr{P}_{i}$ for all $i$.

\section{Safe bounds evaluation for the modal and transition dwell time}

In this section basic set-theoretic techniques to provide bounds for the modal and transition dwell times are discussed.

\subsection{Properties of the modal dwell time}

We begin by showing some some basic properties regarding the dwell times and the invariant sets which can be exploited to find bounds for the modal dwell time.

Definition 3.1 Systems (1) is safe under modal dwell times if there exist a set $\mathscr{X}_{0} \subset \mathscr{X}$ and finite values $\tau_{1}^{*}, \tau_{2}^{*}, \ldots, \tau_{s}^{*}$, such that condition (2) is assured for all initial conditions $x(0) \in \mathscr{X}_{0}$, any $\sigma(0) \in \Sigma$ and for any switching sequence with modal dwell times $\tau_{i} \geq \tau_{i}^{*}$ (for all $i \in \Sigma$ ).

To provide proper lower bounds for the modal dwell times we introduce the quantity

$$
\bar{\tau}_{i} \triangleq \inf \left\{\tau: \Phi_{i}\left(t, \mathscr{P}_{i}\right) \subset \mathscr{P}, \text { for all } t \geq \tau\right\}
$$


Note that, in general, the set appearing on the right hand side of (3) might be empty and consequently the definition might be meaningless. In fact, Definition 2.2 only assures that the trajectories starting in $\mathscr{P}_{i}$ remain in $\mathscr{X}$ but there is no guarantee that they ultimately reach $\mathscr{P}$. It might well happen that, for any initial condition in $\mathscr{P}_{i}, x(t) \notin \mathscr{P}$ for arbitrary large $t$. In this case we set $\bar{\tau}_{i}=+\infty$. With respect to this matter, a result assuring $\bar{\tau}_{i}<+\infty$ is the following.

Proposition $3.1 \bar{\tau}_{i}<+\infty$ if and only if $\bar{x}_{i} \in$ int $\mathscr{P}$.

Proof. (Sufficiency) If $\bar{x}_{i} \in$ int $\mathscr{P}$, then there exists a neighborhood $\mathscr{U}_{\bar{x}_{i}} \subset \mathscr{P}$. Since $\bar{x}_{i}$ is a uniformly stable equilibrium point for the $i$-th dynamics, then there exists $\bar{t}_{i}\left(x_{0}\right)>0$ such that $\Phi_{i}\left(t, \mathscr{P}_{i}\right) \in \mathscr{U}_{\bar{x}_{i}}$ for all $t>\bar{t}_{i}\left(x_{0}\right)$. This means that the value in (3) is bounded.

(Necessity). The necessity comes from the fact that if $\bar{x}_{i} \notin$ int $\mathscr{P}$, then in view of Lemma 2.2, for all $t>\bar{t}_{i}\left(x_{0}\right) \Phi_{i}\left(t, \mathscr{P}_{i}\right)$ includes points which do not belong to $\mathscr{P}$, hence the condition defining the set on the right hand side of Equation (3) cannot be satisfied for finite $\tau$. $\diamond$

Proposition 3.2 If $\bar{x}_{i} \in$ int $\mathscr{P}$ for all $i \in \Sigma$, then system (1) is safe under modal dwell times with $\tau_{i}^{*}=\bar{\tau}_{i}$.

Proof. Let $\mathscr{X}_{0}=\mathscr{P}$. With this choice, any initial condition $x_{0} \in \mathscr{X}_{0}$ is such that $x_{0} \in \mathscr{P}_{i}$, for all $i \in \Sigma$; in particular $x_{0} \in \mathscr{P}_{\sigma(0)}$. This implies, by (3), that any switching sequence with modal dwell times $\tau_{i} \geq \bar{\tau}_{i}$ is such that $x\left(t_{1}\right)=\Phi_{\sigma(0)}\left(t_{1}-t_{0}, \mathscr{P}_{\sigma(0)}\right) \in \mathscr{P}$, hence $x\left(t_{1}\right) \in \mathscr{P}_{\sigma(1)}$, for any $\sigma(1) \in \Sigma$. Repeating this reasoning, it is not difficult to conclude the proof by induction on the switching instants $t_{k}$ and $t_{k-1} . \diamond$

In the next theorem we show that the quantities defined by (3) are indeed lower bounds and they are non-conservative as long as the system is required to evolve in $\mathscr{X}$ for all feasible initial states $x(0) \in \mathscr{P}_{\sigma(0)}$. We remind that such a condition is necessary, according to Proposition 3.1.

Theorem 3.1 The following conditions are equivalent:

i) $x(t) \in \mathscr{X}$ for all $x(0) \in \mathscr{P}_{\sigma(0)}$, for all switching sequences subject to the modal dwell times $\tau_{j}$ and for all $t \geqslant 0$;

ii) $\tau_{j} \geq \bar{\tau}_{j}$, for all $j \in \Sigma$.

Proof. ii) $\Rightarrow$ i). See the last part of the proof of Proposition 3.2.

i) $\Rightarrow$ ii). By contradiction, if there exists $j$ such that $\tau_{j}<\bar{\tau}_{j}$, in view of Lemma 2.2 and (3) there exist $x^{*} \in \mathscr{P}_{j}$ and $t^{*}>\tau_{j}$ such that $\Phi_{j}\left(t^{*}, x^{*}\right) \notin \mathscr{P}$. Therefore, if $\mathscr{P}_{\sigma(0)}=\mathscr{P}_{j}$ and $x(0)=x^{*}$ the evolution of the trajectory is not guaranteed to remain in $\mathscr{X}$ for all $t>t^{*} . \diamond$

The case $\bar{\tau}_{j}=\infty$ means that before switching to another mode an infinite time-interval has to elapse in order to guarantee safety for switching to any of the other modes. Note that $\bar{\tau}_{j}=\infty$ if $\bar{x}_{j} \notin \mathscr{P}_{i}$ for some $i \neq j$ and only if $\bar{x}_{j} \notin i n t \mathscr{P}_{i}$. This basically means that no switching to other modes is allowed, which is a trivial occurrence, as it is equivalent to the system locked to the $j$-th dynamics. However, safety could still be guaranteed by using the concept of transition dwell time, as it is done in the next subsection.

\subsection{Properties of the transition dwell time}

In the previous subsection a theoretical framework has been developed for the modal dwell time; here analogous definitions are introduced and similar results are proven.

Definition 3.2 System (1) is safe under transition dwell times if there exist sets $\mathscr{X}_{i} \subset \mathscr{X}$, for all $i \in \Sigma$ and finite values $\tau_{i j}^{*}$, for all $i \in \Sigma, j \in \Sigma$ and $i \neq j$, such that condition (2) is assured for all initial conditions $x(0) \in \mathscr{X}_{\sigma(0)}$ and for any switching sequence with transition dwell times $\tau_{i j} \geq \tau_{i j}^{*}$ (for all $i \in \Sigma, j \in \Sigma$ and $i \neq j$ ).

Note that this definition refers to several sets of feasible initial conditions, while Definition 3.1 considers a single region of safe initial conditions. The essential reason is that, as we have seen, under modal dwell time, the set $\mathscr{P}$ must be non-empty, being the set of admissible initial condition. Conversely if we consider the transition dwell time the situation is different since the set $\mathscr{P}$ might be empty.

Now, by introducing the quantity

$$
\bar{\tau}_{i j} \triangleq \inf \left\{\tau: \quad \Phi_{j}\left(t, \mathscr{P}_{j}\right) \subseteq \mathscr{P}_{i}, \text { for all } t \geq \tau\right\},
$$

the following results can be proven analogously to the proof of Propositions 3.1 and 3.2.

Proposition 3.3 $\bar{\tau}_{i j}<+\infty$ if and only if $\bar{x}_{j} \in$ int $\mathscr{P}_{i}$, for all $i, j \in \Sigma, i \neq j$.

Proposition 3.4 If $\bar{x}_{j} \in$ int $\mathscr{P}_{i}$ for all $i \in \Sigma, j \in \Sigma, i \neq j$, then system (1) is safe under transition dwell times with $\tau_{i j}^{*}=\bar{\tau}_{i j}$.

Moreover, analogously to Theorem 3.1 the following theorem can be proven.

\section{Theorem 3.2 The following conditions are equivalent}

i) $x(t) \in \mathscr{X}$ for all $x(0) \in \mathscr{P}_{\sigma(0)}$, for all switching sequences subject to the transition dwell times $\tau_{i j}$ and for all $t \geqslant 0$;

ii) $\tau_{i j} \geq \bar{\tau}_{i j}$, for all $i, j \in \Sigma$.

\subsection{Relations among dwell times}

One may wonder if there is any relationship between the modal and the transition dwell time bounds described in the previous subsections. The answer is provided by the following proposition the proof of which follows directly from the definitions of $\bar{\tau}_{i}$ and $\bar{\tau}_{i j}$. 
Proposition 3.5 The following equality holds for all $j: \bar{\tau}_{j}=$ $\max _{i} \bar{\tau}_{i j}$

A lower bound for the safe dwell time may also be defined as

$$
\bar{\tau}=\max _{j} \quad \bar{\tau}_{j}=\max _{i j} \bar{\tau}_{i j}
$$

and an immediate consequence of this definition is the following theorem, reported without proof, which holds when we impose a single dwell time.

Theorem 3.3 The following conditions are equivalent

i) $x(t) \in \mathscr{X}$ for all $x(0) \in \mathscr{P}_{\sigma(0)}$, for all $\sigma(0) \in \Sigma$, for all switching sequences subject to dwell time $\tau$ and for all $t \geqslant 0$

ii) $\tau \geq \bar{\tau}$

\section{The safe transition graph}

In general, the transition between modes based on the global or modal dwell time bounds is not safe if some of the dwell time bounds are infinite. Precisely:

- if $\bar{\tau}=\infty$, violations may occur for some transitions, no matter how long we wait;

- if $\bar{\tau}_{j}=\infty$, violations may occur for some transitions from mode $j$; then $j$ is an "unsafe mode";

- if $\bar{\tau}_{i j}=\infty$ and the active mode is $j$, violations may occur when switching to mode $i$.

A useful tool to check if a transition from one mode to another is safe can be constructed by exploiting the notion of transition weighted graph. Let $G(\Sigma, \mathscr{A})$ be an oriented graph defined as follows. Its nodes correspond to the discrete modes, in simple words $\Sigma$ is the set of nodes. Two nodes $j$ and $i$ are connected if $\tau_{i j}<\infty$. Then the set of arcs is defined as

$$
\mathscr{A}=\left\{(i, j): \quad \bar{\tau}_{i j}<\infty\right\}
$$

Each arch $(i, j)$ from $j$ to $i$, is associated with the weight $\tau_{i j}<\infty$. Then, a simple representation of the transition graph is the matrix

$$
\Theta \triangleq\left[\begin{array}{cccc}
0 & \bar{\tau}_{12} & \ldots & \bar{\tau}_{1 s} \\
\bar{\tau}_{21} & 0 & \ldots & \bar{\tau}_{2 s} \\
: & : & \ldots & : \\
\bar{\tau}_{s 1} & \bar{\tau}_{s 2} & \ldots & 0
\end{array}\right]
$$

According to Proposition 3.3, arc $(i, j)$ exists (i.e. the corresponding entry if finite), if and only if the largest invariant set $\mathscr{P}_{i}$ includes the equilibrium point $\bar{x}_{j}$.

Example 4.1 As an example consider the situation of Figure 2. There are three regions, namely $\mathscr{P}_{1}$ (plain boundary), $\mathscr{P}_{2}$ (dashed boundary), $\mathscr{P}_{3}$ (dotted boundary). Region $\mathscr{P}$

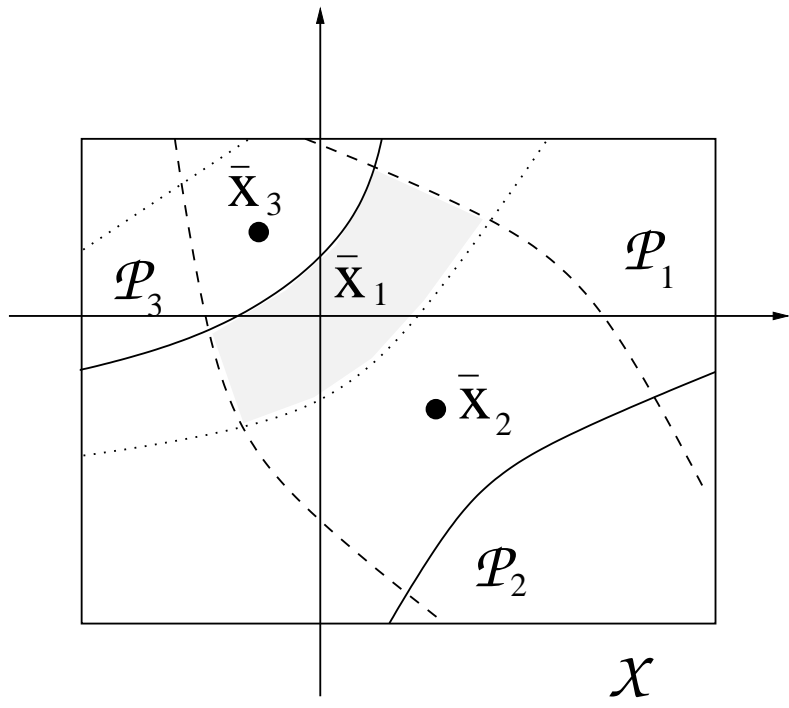

Fig. 2. The regions and their equilibria

is the shaded one. The equilibrium $\bar{x}_{1}=0$ is inside all the regions. The equilibrium $\bar{x}_{2}$ is inside int $\mathscr{P}_{1}$ and outside $\mathscr{P}_{3}$. The equilibrium $\bar{x}_{3}$ is inside int $\mathscr{P}_{2}$ and outside $\mathscr{P}_{1}$. The corresponding matrix $\Theta$ is

$$
\Theta=\left[\begin{array}{ccc}
0 & \bar{\tau}_{12} & \infty \\
\bar{\tau}_{21} & 0 & \bar{\tau}_{23} \\
\bar{\tau}_{31} & \infty & 0
\end{array}\right],
$$

while the resulting graph is sketched in Figure 3.

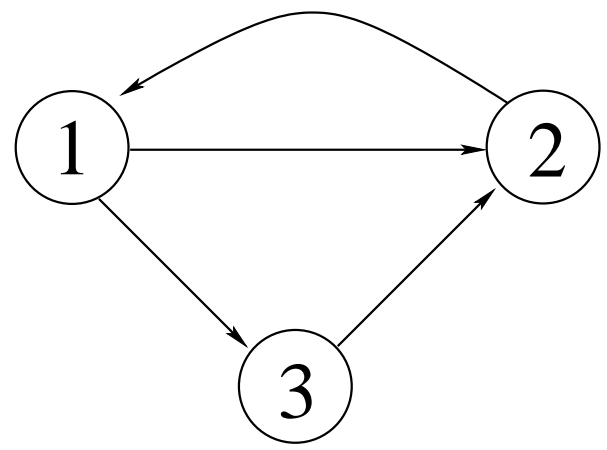

Fig. 3. The graph corresponding to the scenario depicted in Figure

The importance of the transition graph is evidenced in the next definition and theorem.

Definition 4.1 Assume that transition dwell time bounds given by the graph are imposed to the switching sequence. System (1) is transitable iffor any $\sigma(0) \in \Sigma$ and $x(0) \in \mathscr{P}_{\sigma(0)}$ and any $i \in \Sigma$, there exists a sequence $\sigma(\cdot)$ and a time $t<\infty$ such that $\sigma(t)=i$, namely any $i$ can be safely reached. 
For any pair of nodes denote by

$T_{\min }(i, j)$ the value of the shortest path from node $j$ to node $i$.

The following theorem holds.

Theorem 4.1 If the transition graph is connected ${ }^{4}$ then the system is transitable. Furthermore, at most $T_{\min }(i, j)$ time is necessary to reach the discrete mode $i$ from $j$ from each initial condition in $\mathscr{P}_{j}$.

Proof. If $x(0) \in \mathscr{P}_{\sigma(0)}$ then the system is safe if no switching occurs, namely, if $\sigma(t)=\sigma(0)$, for all $t$. However, if $k$ is an adjacent node, after time $\bar{\tau}_{k j}$, the condition (4) is satisfied so a switching to mode $k$ is safe. Since the graph is connected, we can repeat the same reasoning and reach any discrete mode safely. The bounds $T_{\min }(i, j)$ follow immediately. $\diamond$

Remark 4.1 In principle, "safe transitions" could be possible even if the transition graph were not connected. Assume that $\bar{x}_{j}$ is on the boundary of $\mathscr{P}_{i}$ then $\bar{\tau}_{i j}=\infty$. However, for some "special" initial conditions the state, on the mode $\sigma=j$ could converge to $\bar{x}_{j}$ on the "safe side" namely inside $\mathscr{P}_{i}$ so that the commutation to i could be safe. It should be noticed that the condition of the theorem is "almost necessary" in the sense that if we admit the existence of infinitesimal perturbation, then $\bar{x}_{j}$ cannot be on the boundary of $\mathscr{P}_{i}$ for safe commutation.

\subsection{Approximated solutions}

The main problem with the exposed theory is the construction of the transition graph, which requires the determination of the largest invariant sets $\mathscr{P}_{i}$ and the computation of the values $\bar{\tau}_{i j}$. Clearly, approximated solutions are possible if one determines for each dynamic $i$ a proper invariant set $\tilde{\mathscr{P}}_{i}$, not necessarily maximal, including the corresponding equilibrium point $\bar{x}_{i}$. Then lower bounds for the safe dwell times can be found as

$$
\tilde{\tau}_{i} \triangleq \inf \left\{\tau: \Phi_{i}\left(t, \tilde{\mathscr{P}}_{i}\right) \subset \tilde{\mathscr{P}}, \text { for all } t \geq \tau\right\}
$$

where $\tilde{\mathscr{P}}=\bigcap_{j} \tilde{\mathscr{P}}_{j}$ for the modal dwell time and

$$
\tilde{\tau}_{i j} \triangleq \inf \left\{\tau: \quad \Phi_{j}\left(t, \tilde{\mathscr{P}}_{j}\right) \subseteq \tilde{\mathscr{P}}_{i}, \text { for all } t \geq \tau\right\}
$$

for the transition dwell time. For the problem to be well posed, one must assume either

$$
\bar{x}_{i} \in \operatorname{int} \bigcap_{j} \tilde{\mathscr{P}}_{j}, \quad \forall i,
$$

or

$$
\bar{x}_{i} \in \operatorname{int} \tilde{\mathscr{P}}_{j}, \quad \forall i, j
$$

\footnotetext{
4 we remind that an oriented graph is connected for any pair $j, i$ there is an oriented path from $j$ to $i$
}

As a matter of fact, in general, it is not easy to check the conditions (on the trajectory $\left.\Phi_{i}\left(t, \tilde{\mathscr{P}}_{i}\right)\right)$ used in definitions (5) and (6). A possible way to ease the task is to assign the sets $\tilde{\mathscr{P}}_{i}$ as sub-level sets of Lyapunov functions:

$$
\tilde{\mathscr{P}}_{i}=\mathscr{A}_{i} \triangleq\left\{x: \quad \Psi_{i}\left(x-\bar{x}_{i}\right) \leq \alpha_{i}\right\}
$$

where $\Psi_{i}\left(x-\bar{x}_{i}\right)$ is a positive definite smooth function such that, for a proper $\kappa$-function ${ }^{5} \phi_{i}$

$$
\dot{\Psi}_{i}\left(x-\bar{x}_{i}\right) \leq-\phi_{i}\left(\left\|x-\bar{x}_{i}\right\|\right) .
$$

In fact, under the assumptions (7) and (8), in view of (9), one can find proper values $\beta_{i}$, for all $i \in \Sigma$, and $\beta_{i j}$, for all $i \in \Sigma, j \in \Sigma, i \neq j$, such that for all $i \in \Sigma$

$$
\left.\mathscr{B}_{i}=\left\{x: \quad \Psi_{i}\left(x-\bar{x}_{i}\right) \leq \beta_{i}\right\} \subset \bigcap_{j}\right\} \mathscr{A}_{j}
$$

and, for all $i, j \in \Sigma, i \neq j$

$$
\mathscr{B}_{i j}=\left\{x: \quad \Psi_{j}\left(x-\bar{x}_{j}\right) \leq \beta_{i j}\right\} \subset \mathscr{A}_{i}
$$

Once these values are found, one can find balls $\mathscr{R}_{i}$ and $\mathscr{R}_{i j}$ with centers $\bar{x}_{i}$ and radius $\rho_{i}$ and $\rho_{i j}$, respectively, such that

$$
\mathscr{R}_{i}=\left\{x:\left\|x-\bar{x}_{i}\right\| \leq \rho_{i}\right\} \subset \mathscr{B}_{i}, \quad \text { for all } i \in \Sigma
$$

and

$$
\begin{array}{r}
\mathscr{R}_{i j}=\left\{x:\left\|x-\bar{x}_{j}\right\| \leq \rho_{i j}\right\} \subset \mathscr{B}_{i j}, \\
\text { for all } i \in \Sigma, j \in \Sigma, i \neq j .
\end{array}
$$

It is not difficult to see that proper approximate bounds can be found as follows.

Proposition 4.1 Assume that the conditions (7) holds true. Then for all $i \in \sum$ there exist values $\beta_{i}$ satisfying (10) and there exist $\rho_{i}$ satisfying (12). Then

$$
\tilde{\tau}_{i}=\frac{\alpha_{i}-\beta_{i}}{\phi_{i}\left(\rho_{i}\right)}
$$

is a safe safe lower bounds for the $i$-th modal dwell time. Proof. It follows easily from the fact that outside $\mathscr{B}_{i}$ the Lyapunov derivative is $\dot{\Psi}_{i}\left(x-\bar{x}_{i}\right) \leq-\phi_{i}\left(\rho_{i}\right)$, then

$$
\Psi_{i}\left(x(t)-\bar{x}_{i}\right) \leq-\phi_{i}\left(\rho_{i}\right) t+\Psi_{i}\left(x(0)-\bar{x}_{i}\right) \leq-\phi_{i}\left(\rho_{i}\right) t+\alpha_{i}
$$

for all $x(0) \in \mathscr{A}_{i}$. Hence, if $x(0) \in \mathscr{A}_{i}$ and $\sigma(t)=i$ for all $t \in\left[0, \tilde{\tau}_{i}\right), x\left(\tilde{\tau}_{i}\right) \in \mathscr{B}_{i} . \diamond$

Analogously, the following result can be proven.

\footnotetext{
5 a $\kappa$-function $\phi(\xi), \xi \geq 0$, is a strictly increasing continuous function with $\phi(0)=0$.
} 
Proposition 4.2 Assume that the conditions (8) holds true. Then for all $i \in \Sigma, j \in \Sigma, i \neq j$, there exist values $\beta_{i j}$ satisfying or(11), there exist $\rho_{i j}$ satisfying (13) and

$$
\tilde{\tau}_{i j}=\frac{\alpha_{j}-\beta_{i j}}{\phi_{j}\left(\rho_{i j}\right)} .
$$

is a safe lower bounds for the transition dwell time from $i$ to $j$.

\section{Reset Maps and mode-dependent constraints}

An interesting generalization of the presented theory is the case in which reset maps are assigned. Assume that the map $\Psi_{i j}(x)$, defined on $\mathscr{X}$, is assigned for the $j$-to $-i$ transition so that if $\sigma(t)=j$ for $t<t_{k}$ and $\sigma(t)=i$ for $t>\geq t_{k}$ then

$$
x\left(t_{k}^{+}\right)=\Gamma_{i j}\left(x\left(t_{k}^{-}\right)\right)
$$

We assume that $\Psi_{i j}$ are continuous and invertible. Given a compact set $\mathscr{C}$, define the pre-image as

$$
\Psi_{i j}^{-1}(\mathscr{C}) \doteq\left\{x: \Psi_{i j}(x) \in \mathscr{C}\right\}
$$

which is also a compact set. Then the modal and transition dwell times can be computed as follows

$$
\begin{gathered}
\bar{\tau}_{j} \triangleq \inf \left\{\tau: \Phi_{j}\left(t, \mathscr{P}_{j}\right) \subset \Psi_{i j}^{-1}(\mathscr{P}), \text { for all } t \geq \tau\right\} \\
\bar{\tau}_{i j} \triangleq \inf \left\{\tau: \Phi_{j}\left(t, \mathscr{P}_{j}\right) \subseteq \Psi_{i j}^{-1}\left(\mathscr{P}_{i}\right), \text { for all } t \geq \tau\right\},
\end{gathered}
$$

It is not difficult to see that the necessary and sufficient conditions for the well posedness in this case are

$$
\bar{x}_{j} \in \operatorname{int} \Psi_{i j}^{-1}(\mathscr{P})
$$

and

$$
\bar{x}_{j} \in \operatorname{int} \Psi_{i j}^{-1}\left(\mathscr{P}_{i}\right)
$$

respectively. All the developed theory remains essentially unchanged.

As a further extension of interest consider the case of modedependent constraints, namely

$$
x(t) \in \mathscr{X}_{i}, \quad \text { if } \sigma(t)=i
$$

In this case the theory works as well with the only change that the sets $\mathscr{P}_{i}$ are the largest invariant sets in $\mathscr{X}_{i}$.

\section{Systems with affine dynamics}

A case of practical interest in which "almost exact solutions" can be found, is that of systems with piecewise affine dynamics

$$
\dot{x}(t)=A_{\sigma(t)} x(t)+b_{\sigma(t)}
$$

where the $A_{i}$ 's are Hurwitz matrices and the $b_{i}$ 's are given vectors for all $i \in \Sigma$. Assume that the set $\mathscr{X}$ is a convex and compact polyhedron including the equilibrium vectors:

$$
\bar{x}_{i}=-A_{i}^{-1} b_{i} \in \operatorname{int} \mathscr{X} .
$$

Assume also that reset square invertible matrices $R_{i j}$ are assigned for the $j-$ to $-i$ commutation. In this case, sets $\mathscr{P}_{j}$ are compact and convex and have non-empty interiors. Therefore [4] each set $\mathscr{P}_{k}$ can be arbitrarily internally approximated by a positively invariant polyhedral set $\hat{\mathscr{P}}_{j}$. The computation involve the Euler Auxiliary System (EAS)

$$
x(k+1)=\left[I+\tau A_{i}\right] x(k)+\tau b_{i}
$$

and the technique proposed in [11]. According to [11], if $F x \leq g$ is the "plane description of $\mathscr{X}$, then the set $\hat{\mathscr{P}}_{i}$ is identified by the inequalities

$$
F\left[I+\tau A_{i}\right]^{k}\left(x-\bar{x}_{i}\right) \leq g, \quad k=0,1, \ldots, K_{i}
$$

for some finite $K_{i}$. According to [4] this set is the largest invariant for the (EAS) within $\mathscr{X}$, it is positively invariant for the continuous-time system, and for $\tau \rightarrow 0$ it converges to $\hat{\mathscr{P}}_{i}$.

Proposition 6.1 Assume that the necessary and sufficient conditions $\bar{x}_{i} \in$ int $\mathscr{P}$ (respectively $\bar{x}_{i} \in$ int $\mathscr{P}_{j}$ ) are satisfied. Then these conditions are met by suitable polyhedral approximating sets, precisely $\bar{x}_{i} \in$ int $\hat{\mathscr{P}}$ (respectively $\bar{x}_{i} \in$ int $\hat{\mathscr{P}}_{j}$ ).

Henceforth we assume, without restrictions, that $\mathscr{P}_{i}$ are polytopes and that $\bar{x}_{i}$ satisfy the above conditions. By using this approximation, an algorithm to determine the optimal values for the modal and transition dwell times and, therefore, the transition graph, can be designed. The basic problem to be faced when determining the quantities defined in (3) and (4) is to generically compute

$$
\bar{\tau}=\inf \left\{\tau: \Phi(t, \mathscr{P}) \subset R^{-1} \mathscr{Q} \text { for all } t \geq \tau\right\}
$$

for given polytopes $\mathscr{P}$ and $\mathscr{Q}$ and a given reset matrix $R$, where

$$
\Phi(t, \mathscr{P})=e^{A t} \mathscr{P} .
$$

Let $p_{k}, k=1,2, \ldots, v_{\mathscr{P}}$ be the vertices of $\mathscr{P}$ and $q_{h} x \leq$ $r_{h}, h=1,2, \ldots$, the inequalities representing $\mathscr{Q}$. Then the following procedure computes $\bar{\tau}$.

\section{Procedure 6.1}

(1) $\bar{\tau}:=0$.

(2) For $k=1,2, \ldots, v_{\mathscr{P}}$ (each vertex):

(a) For each $h=1,2, \ldots, v_{\mathscr{Q}}$ (each plane): compute the value

$$
\theta:=\min \left\{\tau: q_{h} R e^{A t} v_{k} \leq r_{k}, \text { for all } t \geq \tau\right\}
$$


(b) If $\bar{\tau}<\theta$ then $\bar{\tau}:=\theta$.

Procedure 6.1 produces, in principle, non-conservative solutions since by means of polytopes we can arbitrarily approximate the largest invariant set. However, the complexity of the description of the derived polytopic invariant sets can be arbitrarily large. As it is known, approximate solutions can be always found by considering ellipsoidal invariant sets. A trivial solution is quite simple and sounds as follows. For each $\bar{x}_{i}$ we take an ellipsoid

$$
\mathscr{E}_{i}=\left\{x:\left(x-\bar{x}_{i}\right)^{T} P_{i}\left(x-\bar{x}_{i}\right) \leq 1\right\} \subset \mathscr{X}
$$

which is invariant for the $i$ translated dynamics

$$
\frac{d}{d t}\left(x-\bar{x}_{i}\right)=A_{i}\left(x-\bar{x}_{i}\right)=A_{i} x+b_{i}
$$

and the corresponding quadratic functions according to the results of Section 4.1. Precisely the transition from $j$ to $i$ is enabled if $x(t) \in \mathscr{E}_{i}$.

A less conservative solution can be found by resorting to composite quadratic Lyapunov functions [14], by associating different ellipsoids to different transitions.

$$
\mathscr{E}_{i j}=\left\{x:\left(x-\bar{x}_{i}\right)^{T} P_{i j}\left(x-\bar{x}_{i}\right) \leq 1\right\} \subset \mathscr{X}
$$

and such that $\bar{x}_{j} \in \operatorname{int} \mathscr{E}_{i j}$.

The following proposition holds.

Proposition 6.2 Assume that for every $i$ and $j \mathscr{E}_{i j}$ are found which have the following properties

p1) they are invariant for (18);

p2) they are included in $\mathscr{X}$;

Then for $\sigma(0)=i$ and $x(0) \in \operatorname{conv}_{j} \mathscr{E}_{i j}$, where "conv $v_{j}$ denotes the convex hull of the union, the system is safe under appropriate transition dwell times $\bar{\tau}_{i j}<\infty$.

The proof is quite trivial and omitted. Rather, let us consider an ellipsoid $x^{T} Q^{-1} x \leq 1$, with $Q$ positive definite, a linear system $\dot{x}=A x$, a point $z$ and let $\mathscr{X}=\left\{x: f_{i}^{T} x \leq 1, \quad i=\right.$ $\left.1, \ldots, v_{\mathscr{Q}}\right\}$. The inclusion of the ellipsoid in $\mathscr{X}$, its invariance and the inclusion of the point $z$ in the ellipsoid, can be expressed as

$$
\begin{aligned}
& f_{i}^{T} Q f_{i} \leq 1, \quad i=1, \ldots, v_{\mathscr{Q}} \\
& A Q+Q A^{T}<0 \\
& z^{T} Q^{-1} z<1
\end{aligned}
$$

It is easy to show that conditions (20)-(21) are convex in $Q$, and so finding $Q>0$ is efficiently solvable.
Remark 6.1 If we consider the convex hull of the ellipsoids conv $_{i} \mathscr{E}_{i j}$ this is obviously invariant for (18). The corresponding induced function, positively homogeneous of the second order is a composite quadratic function as proposed in [14]. Note that the one considered here is a special case since in general the generating ellipsoids do not need to be separatedly invariant as in our case.

\section{Stability under dwell time}

A special case of the affine problem is that in which all the systems share a common equilibrium point $\bar{x}_{j}=\bar{x}=0$. In this case safety is equivalent to stability. Consider the system

$$
\dot{x}(t)=A_{\sigma(t)} x(t) .
$$

According to the theory developed in the previous section, conditions for the state of the system to be included in a given 0 -symmetric polytopic set $\mathscr{X}$ can be established. With little additional effort, asymptotic stability can be assured as well. Assume, without restrictions, that $\mathscr{P}_{i}$ is also a 0 symmetric polytope.

To ensure asymptotic stability it is sufficient to introduce an arbitrarily small contraction factor $0<\lambda<1$ and define

$$
\bar{\tau}_{i} \triangleq \inf \left\{\tau: e^{A_{i} t} \mathscr{P}_{i} \subset \lambda \mathscr{P}, \text { for all } t \geq \tau\right\}
$$

and

$$
\bar{\tau}_{i j} \triangleq \inf \left\{\tau: e^{A_{j} t} \mathscr{P}_{j} \subset \lambda \mathscr{P}_{i}, \text { for all } t \geq \tau\right\}
$$

The following theorem is the concluding result of the paper. Its proof is conceptually easy but tedious, hence it is not reported.

Theorem 7.1 Under the modal (resp. transition) dwell times computed by means of (23) (resp. (24)) and by means of Procedure 6.1 system (22) is asymptotically stable.

\subsection{Absolute, modal and transition dwell time: some com- ments}

As a general remark note that seeking for a single dwell time instead of modal or transition dwell times can be very restrictive. We show this fact by means of a simple example. Consider again the family (22) and suppose that

$$
A_{i}=\xi_{i}\left[\begin{array}{cc}
-\mu_{i} & -a_{i} \\
1 / a_{i} & -\mu_{i}
\end{array}\right]
$$

with $a_{1}=1 / 4, a_{2}=4$ and $\mu_{i}$ and $\xi_{i}$ positive, whose trajectories are reported in Figure 4. The first dynamics are associated with $\mathscr{P}_{1}$, the "vertical" ellipse while the second with $\mathscr{P}_{2}$, the "horizontal" one. Denote by $\tau_{21}$ the time required for $\Phi\left(t, \mathscr{P}_{1}\right)$ to ultimately enter $\mathscr{P}_{2}$ and $\tau_{12}$ the time 
required for $\Phi\left(t, \mathscr{P}_{2}\right)$ to ultimately enter $\mathscr{P}_{1}$. It is not difficult to see that if $\xi_{1} \ll \xi_{2}$, then $\tau_{12} \gg \tau_{12}$. Thus taking a common dwell time might be quite penalizing. On the other hand, if $\mu_{2}$ is small, namely the state associated with the second dynamics rotates quickly but it is poorly damped, in practice, to assure stability by means of a single dwell time one needs to take $\tau \approx \tau_{21}$.

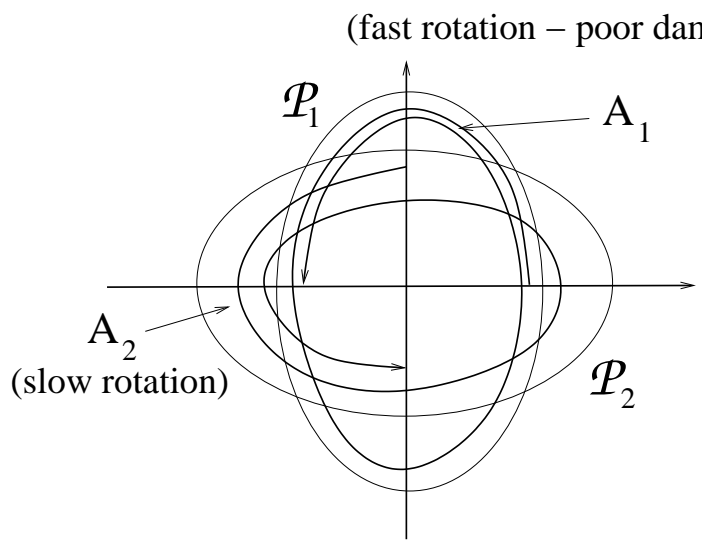

Fig. 4. The trajectories for system (25).

\section{Example}

We conclude the paper with the following numerical example. Consider the circuit depicted in Figure 5 where $L=$ $1 \mathrm{mH}, \mathrm{C}=1 \mathrm{mF}$ and $\bar{V}=0.5 \mathrm{~V}$. The system can switch in two ways: between configuration (a) and configuration (b) and in each of these it can switch the resistance value: $R_{1}=10 \Omega$, $R_{2}=100 \Omega, R_{3}=100 \mathrm{~m} \Omega, R_{4}=10 \mathrm{~m} \Omega$. The following four configurations are possible.

\begin{tabular}{|c|c|c|c|}
\hline$\Sigma_{1}$ & $\Sigma_{2}$ & $\Sigma_{3}$ & $\Sigma_{4}$ \\
\hline (a) with $R_{1}$ & (a) with $R_{2}$ & (b) with $R_{3}$ & (b) with $R_{4}$ \\
\hline
\end{tabular}

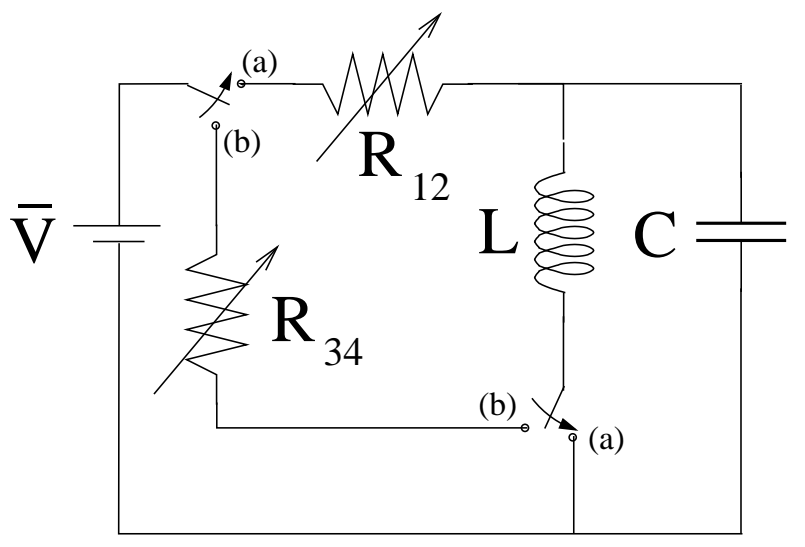

Fig. 5. The switching circuit used in Section 8.

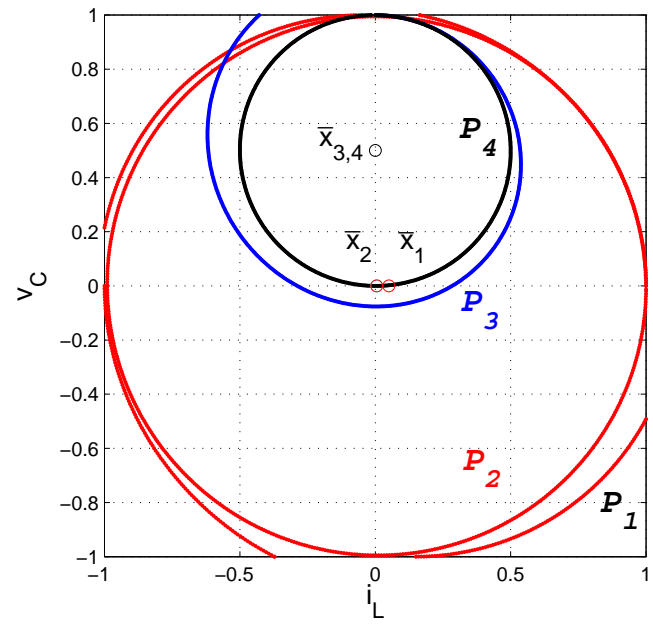

Fig. 6. Maximal invariant regions for the circuit in Figure 5.

Denoting by $x(t)=\left[i_{L}(t) v_{C}(t)\right]^{T}$ the state variable, the state equations are

$$
\Sigma_{1,2}: \dot{x}=\left[\begin{array}{cr}
0 & \frac{1}{L} \\
-\frac{1}{C} & -\frac{1}{R_{1,2} C}
\end{array}\right] x+\left[\begin{array}{c}
0 \\
\frac{1}{R_{1,2} C}
\end{array}\right] \bar{V}
$$

for the configuration (a) and

$$
\Sigma_{3,4}: \dot{x}=\left[\begin{array}{rr}
-\frac{R_{3,4}}{L} & -\frac{1}{L} \\
\frac{1}{C} & 0
\end{array}\right] x+\left[\begin{array}{c}
\frac{1}{L} \\
0
\end{array}\right] \bar{V}
$$

for the switch configuration (b). The corresponding equilibrium points are

$$
\bar{x}_{1,2}=\left[\begin{array}{c}
\bar{V} \\
R_{1,2} \\
0
\end{array}\right] \quad \bar{x}_{3,4}=\left[\begin{array}{c}
0 \\
\bar{V}
\end{array}\right]
$$

The largest invariant sets contained in the set $\mathscr{X}=\left\{|x|_{\infty} \leq\right.$ 1) for the different modes are depicted in Figure 6 along with the steady state values (circles).

From the calculated regions and steady state values it turns out that $\bar{x}_{2}$ belongs to every maximal invariant set (though it is very close to the boundary of $\mathscr{P}_{4}$ ), whereas $\bar{x}_{1}$ does not belong to $\mathscr{P}_{4}$, as evidenced in the resulting transition matrix (time in $m s$ )

$$
\Theta=\left[\begin{array}{cccc}
0 & 0 & 0.2 & 0 \\
1.9 & 0 & 2.0 & 0.2 \\
50.7 & 524 & 0 & 0 \\
+\infty & 1969 & 4.6 & 0
\end{array}\right]
$$




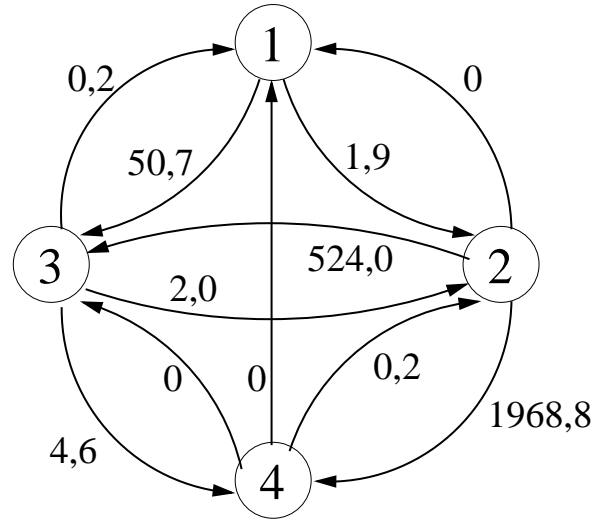

Fig. 7. Transitions graph for the circuit in Figure 5.

corresponding to the transition graph in Figure 7 . The corresponding dwell-time vector is

$$
\left[\begin{array}{llll}
\infty & 1968 & 4.6 & 0.2
\end{array}\right]
$$

which means that no modal dwell time exist which assure safe transition from the first mode, which is motivated by the fact that $\bar{x}_{1} \notin \mathscr{P}_{4}$.

\section{Conclusion}

The problem of a switching plant in the presence of constraints has been investigated. The dynamics involved are assumed stable but they are possibly associated with different equilibria. We have proven that an efficient way to meet the constraints is to compute the modal and the transition dwell time, which are less restrictive then the standard global dwell time. We have presented a set-theoretic methods for the computation and we have shown that the transition dwell time leads to the concept of dwell-time graph which allow safe transitions between modes. The switching limiter is proposed as an application. In the case of systems with affine dynamics, a computation algorithm based on polyhedral computation has been presented. Stability under switching is analyzed as special case.

\section{References}

[1] D. Angeli and E. Mosca, "Lyapunov-based switching supervisory control of nonlinear uncertain systems", IEEE Trans. Automat. Control, vol. 47, no. 3, pp. 500-505, 2002.

[2] P. Antsaklis and H. Lin, "Stability and stabilizability of switched linear systems" IEEE Trans. Automat. Control, vol. 58, no. 2, 2009.

[3] F. Blanchini and C. Savorgnan, "Stabilizability of switched linear systems does not imply the existence of convex Lyapunov functions", Automatica, vol. 44, no. 4, pp. 1166-1170, April 2008.

[4] F. Blanchini and S. Miani, "Constrained stabilization of continuoustime linear systems", Systems Control Lett., vol. 28, no. 2, pp. 95 102,1996

[5] F. Blanchini, F. A. Pellegrino and L. Visentini, "Control of manipulators in a constrained workspace by means of linked invariant set”, International Journal on Robust and Nonlinear Control, vol 14 pp. $1185-1505,2004$.
[6] F. Blanchini and S. Miani, "Set theoretic methods in control", Birkhauser, Boston, 2008.

[7] M. S. Branicky, "Multiple Lyapunov functions and other analysis tools for switched and hybrid systems," IEEE Trans. Automat. Control, vol. 43, no. 4, pp. 475-482, 1998.

[8] P. Colaneri and J.C. Geromel " $\mathscr{H}$ and dwell time specification of switched linear systems", Proceedings of the 47th Conference on decision and Control, Cancun, pp. 5318-5323, Dec. 2008,.

[9] G. Chesi, P. Colaneri, J.C. geromel, R. Middleton, and R. Shorten, "On the minimum dwell time for linear switching systems", submitted.

[10] R. A. Decarlo, M. S. Branicky, S. Pettersson and B. Lennartson, "Perspectives and results on the stability and stabilizability of hybrid systems," Proceedings of the IEEE, vol. 88, no. 7, pp. 1069-1082, 2000.

[11] E. G. Gilbert and K. K. Tan, "Linear systems with state and control constraints: the theory and the applications of the maximal output admissible sets", IEEE Trans. Automat Control, vol. 36, no. 9, pp. 1008-1020, 1991.

[12] J. J.P. Hespanha and A. S. Morse, "Stability of switching systems with average dwell time", Proceedings of the 38 CDC, pp. 26552660, 1999.

[13] J.P. Hespanha "Uniform Stability of switched Linear Systems: Extension of LaSalle's Invariance Principle", IEEE Trans. Automat Control, vol. 49, no. 4, April 2004.

[14] T. Hu and Z. Lin, "Composite quadratic Lyapunov functions for constrained control systems," IEEE Trans. on Automat. Contr., Vol.48, No. 3, pp.440-450, 2003.

[15] I.V. Kolmanovski and E.G. Gilbert. "Multimode regulators for systems with state and control constraints and disturbance input", In A.S. Morse, editor, Lecture Notes in Control and Inform. Sci, 222, Control using logic-based switching, pp. 104-117, Springer-Verlag Ltd., London, 1997.

[16] D. Liberzon, Switching in System and Control, ser. Systems and Control: Foundations \& Applications. Boston: Birkhauser, 2003.

[17] D. Liberzon and A. S. Morse, "Basic problems in stability and design of switched systems," IEEE Contr. Syst. Mag., vol. 19, no. 5, pp. 59-70, 1999.

[18] M. W. McConley, B.D. Appleby, M. A. Dahleh and E. Feron, "A computationally efficient Lyapunov-based scheduling procedure for control of nonlinear systems with stability guarantees", IEEE Trans. Automat Control, vol. 45, no. 1, pp. 33-49, 2000.

[19] A. S. Morse, "Supervisory control of families of linear set-point controllers. I: Exact matching," IEEE Trans. Automat. Control, vol. 41, no. 10, pp. 1413-1431, 1996.

[20] _ - "Supervisory control of families of linear set-point controllers. II: Robustness," IEEE Trans. Automat. Control, vol. 42, no. 11, pp. 1500-1515, 1997.

[21] R. Shorten, F. Wirth, O. Mason, K. Wulff, C. King, "Stability Criteria for Switched and Hybrid Systems". SIAM review, vol. 49, no. 4, pp. 545-592, 2007.

[22] Z. Sun and S. Ge, Switched Linear Systems Control and Design, ser. Communications and Control Engineering. London: SpringerVerlag, 2005.

[23] G.F. Wredenhagen and P.R. Belanger, "Piecewise-linear LQ control for systems with input constraint", Automatica J. IFAC, vol. 30, no. 3, pp. 403-416, 1994. 